\title{
TEACHING NOTE: THE ONE-QUESTION POP QUIZ
}

\author{
Anne Hadley Behrend \\ Liberty University
}

Most students believe the unannounced quiz to be a veritable monster lurking in the shadows of their academic careers. The One-Question Quiz model of the beast, however, they actually enjoy, and that is just one of the beauties of the strategy.

When preparing the syllabus for the start of a first-year college history course, Advance Placement (AP), or International Baccalaureate (IB) history class, one requirement stands out from the start: These young people are going to be asked to read an enormous amount. Although many challenges await the students who will grapple with the rigors of these demanding courses, the sheer number of words they must absorb is the most daunting. How can we teachers make turning the pages of their history text and supplementary readings more alluring? Part of the answer can be an appealing little tool known as the One-Question Quiz.

After choosing a lively text that tells the story of the world's past, a book with authority, yes, but also with vitality and even humor, the teacher must be sure her enthusiasm for the volume shines through as she introduces the text to her students. And she must emphasize the importance of keeping up with each day's reading, in order to avoid the pitfalls of procrastination.

But in the real world, these young readers lead busy lives, full of academics, sports, family activities, social life, and, for many of them, jobs. So it becomes easy to think skipping the assignment for one day won't really hurt anything until the work gets out of hand, at which point the student is overwhelmed with the fear of never being able to catch up.

Several strategies are available to motivate highly grade-conscious students who sign up for these courses. The most effective method I have found is one I hit upon when searching for something pedagogically effective while also fun for my students: The One-Question Quiz. The beauties of the One-QQ are several: It teaches as well as it tests; it is quick; and it is popular with students in the class.

The One-QQ is always based on the reading assignment from the night before. The quiz consists, as advertised, of one multiple choice question. The item has five possible answers to choose from: A, B, C, D, and E. The primary reason for using this format is that it echoes the design of the multiple choice questions on the AP exam, and in fact I often use questions from past AP exams published by the College Board. Thus, students are not only learning the information covered by the quiz, but they are getting practice in the rigor of AP-type questions.

After printing the question I have typed on the computer, I cut the pages into strips just large enough for the question stem and five answer choices, plus a small space for the student's name. I also make a simple PowerPoint slide showing the same question and answer choices. I never announce the dates of the quizzes, of course, but 
the class knows they can expect them every few days. It is important to occasionally follow a quiz with another within the next day or two so the class will not be able to predict their frequency.

As soon as class starts on the day I have a One-QQ ready, I hold up the packet of question strips and ask students to clear their desks and have pencil or pen at the ready. Pseudo-groans are heard around the room, but there are grins, too, and sparkling eyes from those who have done the previous night's reading. From the one or two who have not read the assignment, the groans are real!

I pass out the question strips quickly, asking the students to mark their answers promptly, add their names to the slips, and then fold them. I give everyone a minute or so to check their answers and then collect the slips, starting with the students who got them first. The whole process takes no more than two or three minutes. No one tries to copy his neighbor's slip. Students are quite careful, in fact, to conceal their choice of answer and get their question strip folded.

Immediately, the whole class discusses which is the correct answer and why. I project the PowerPoint slide onto a screen so that everyone can see the stem and answer choices. We talk about the substance of the facts covered by the question and we point out why the other answers are wrong. Then we speak about strategies for guessing if a student is not sure of an answer.

The principal reason that learners like these quizzes is because of the grading system I use. If someone has marked the correct answer, he or she earns a "daily" A. However, if the question is marked incorrectly, the student gets nothing - no grade at all. Therefore, if students do the reading they can accumulate a string of "daily" A's which are averaged with other daily grades at the end of the marking period. That average becomes a major grade. Members of the class are not penalized if they miss the question: It doesn't help them, but it doesn't hurt them either.

Most students choose the correct answer on the majority of One-QQ's. They increase their overall grade, they learn facts and concepts of the subject more securely, and they become comfortable with the format of AP multiple choice questions. Also, it adds a spark to the class for that day.

At the year's end, when filling out course evaluation sheets, in the space for strengths of the class, almost without exception students write some version of "OneQuestion Quizzes-they're awesome!" 